\title{
Interval valued neutrosophic sets and multi-attribute decision-making based on generalized weighted aggregation operator
}

\author{
Zhao Aiwu ${ }^{\mathrm{a}, *}$, Du Jianguo ${ }^{\mathrm{a}}$ and Guan Hongjun ${ }^{\mathrm{b}}$ \\ ${ }^{a}$ School of Management, Jiangsu University, Zhenjiang, P.R. China \\ ${ }^{\mathrm{b}}$ School of Management Science and Engineering, Shandong University of Finance and Economics, Jinan, P.R. China
}

\begin{abstract}
Neutrosophic sets are powerful logics designed to facilitate understanding of indeterminate and inconsistent information; many types of incomplete or complete information can be expressed as interval valued neutrosophic sets (IVNSs). This paper proposes improved aggregation operation rules for IVNSs, and extends the generalized weighted aggregation (GWA) operator to work congruently with IVNS data. The aggregated results are also expressed as IVNSs, which are characterized by truthmembership degree, indeterminacy-membership degree, and falsity-membership degree. The proposed method is proved to be the maximum approximation to the original data, and maintains most of the information during data processing. Then, a method is proposed to determine the ranking orders for all alternatives according to their comparative advantage matrices based on their general score, degree of accuracy and degree of certainty expressed by the aggregated IVNSs. Finally, a numerical example is provided to illustrate the applicability and efficiency of the proposed approach.
\end{abstract}

Keywords: Interval valued neutrosophic sets, multi-attribute decision making, generalized weighted aggregation, fuzzy sets

\section{Introduction}

Due to the complexity and uncertainty of objective things, and the ambiguity of human thinking, Zadeh proposed a remarkable theory of fuzzy sets (FSs) in 1965 [13]. Since then, the FS theory has been successfully applied to various fields of multi-attribute decision-making. Moreover, extended FSs were developed, such as the interval valued fuzzy sets (IVFSs) [5], intuitionistic fuzzy sets (IFSs) [12], interval valued intuitionistic fuzzy sets (IVIFSs) [11], hesitant fuzzy sets (HFSs) [18], etc. Although FS theory has been developed and generalized, it cannot account for all possible uncertainties in a variety of physical problems. For

\footnotetext{
*Corresponding author. Zhao Aiwu, School of Management Jiangsu University, No 301, Xuefu Road, Zhenjiang, P.R. China. Tel./Fax:+86 511 88790922; E-mail: Aiwuzh@126.com.
}

instance, when an expert is asked a question, he or she may think that the possibility of a true answer is equal to 0.6 , the possibility of a false answer is 0.4 , and the degree of their uncertainty is 0.2. This issue is beyond the scope of FSs and IFSs. Hence, Smarandache proposed neutrosophic logic and neutrosophic sets (NSs) in 1999 [1]. A NS is a set in which each element of the universe has respective degrees of truth, indeterminacy, and falsity, which lie in the nonstandard unit interval of ] $0^{-}, 1^{+}[$. This method represents an extension of the standard interval $[0,1]$ used for IFSs. The uncertainty presented here, (i.e., the indeterminacy factor) is independent of the truth and falsity values. This extended IFS theory to account for uncertain information.

Many scholars have begun to study the practical application of NSs in multi-attribute decision-making. Wang, et al. [2] and Ye [9] defined the aggregation operators for single valued neutrosophic set (SNSs), 
and proposed a corresponding multi-attribute decisionmaking method. Majumdar, et al. [14] defined the distance, similarity and entropy of two SNSs. Ye $[7,10]$ defined the Hamming distance of SNSs based on crossentropy, and proposed a corresponding multi-attribute decision-making method. Based on the operators of SNSs, Wand and Li [6] extended the Todim method based on multi-valued neutrosophic sets. Wang, et al. [3] defined IVNSs and their logic operation rules. Ye [8] defined similarity measure operators based on the Hamming and Euclidean distances, and applied them to multi-attribute decision-making problems. Broumi, et al. [17] defined the correlation coefficient of IVNSs. Chi, et al. [15] extended the TOPSIS method to include multi-attribute decision programs with unknown weights and IVNS values. Zhang, et al. [4] defined the operation rules for IVNSs based on T-norm and S-norm, and proposed a corresponding multi-attribute decision-making method.

All of the aforementioned definitions of aggregation operators observe certain rationalities, but some are inconsistent with the reality logic. Due to the realistic differences among the characteristics of truth-membership degree, indeterminacy-membership degree, and falsity-membership degree, operation rules must not only consider the basic operation rules, but also consider the realistic significance of operators. In many multi-attribute decision-making applications, the weight of each attribute is determined according to its importance. The generalized weighted aggregation operator is utilized to obtain the aggregated value for all attributes of each alternative, and rank the limited alternatives or select the best by comparing the advantage degrees of the aggregated IVNSs representing each alternative. This paper proposes improved operation rules for IVNSs, and defines the generalized weighted aggregation operator for IVNSs based on a traditional GWA operator. Due to the maximum approximation to original values, the aggregated value based on the GWA operator can most effectively reflect the multi-attribute values while maintaining the greatest amount of information. Compared to the multi-attribute decision-making method based on distance, this method maintains the integrity of information during the calculation process. Meanwhile, by choosing different parameters, decision makers can obtain ranking order results according to their own risk attitudes.

The rest of this paper is organized as follows. In Section 2, some concepts and operations of IVNSs are briefly introduced. Based on the basic operation rules, the GWA operator is extended to IVNSs. In
Section 3, a decision-making method is developed for IVNSs based on a GWA operator. In Section 4, an example is presented to illustrate the proposed method, and comparison analysis and discussion are provided. Finally, Section 5 concludes the paper.

\section{IVNS concepts and operators}

Definition 1. [8] Let $\mathrm{X}$ be a space of points (objects) with generic elements in $X$, denoted by $x$. An interval valued neutrosophic set (IVNS) $A$ in $X$ is characterized by truth-membership function $T_{A}(x)$, indeterminacymembership function $I_{A}(x)$ and falsity-membership function $F_{A}(x)$. For each point $x$ in $X, T_{A}(x), I_{A}(x)$, $F_{A}(x) \in[0,1]$.

$$
\begin{aligned}
A= & \left\{<x,\left[T_{A}^{l}(x), T_{A}^{r}(x)\right],\left[I_{A}^{l}(x), I_{A}^{r}(x)\right],\right. \\
& {\left.\left[F_{A}^{l}(x), F_{A}^{r}(x)\right]>\mid x \in X\right\} }
\end{aligned}
$$

An IVNS is also defined as $A=\left\langle\left[T_{A}^{l}, T_{A}^{r}\right]\right.$, $\left.\left[I_{A}^{l}, I_{A}^{r}\right],\left[F_{A}^{l}, F_{A}^{r}\right]\right\rangle$.

Definition 2. The complement of an interval valued neutrosophic set $A=\left\langle\left[T_{A}^{l}, T_{A}^{r}\right],\left[I_{A}^{l}, I_{A}^{r}\right],\left[F_{A}^{l}, F_{A}^{r}\right]\right\rangle$ is defined by

$$
A^{c}=\left\langle\left[F_{A}^{l}, F_{A}^{r}\right],\left[I_{A}^{l}, I_{A}^{r}\right],\left[T_{A}^{l}, T_{A}^{r}\right]\right\rangle
$$

The maximum of an IVNS is $<[1,1],[0,0],[0,0]>$, and the minimum is $<[0,0],[0,0],[1,1]\rangle$. This definition varies from the definition of IVNSs presented in previous literature; when a cost IVNS is transformed into a benefit IVNS, the truth-membership and falsitymembership are altered, while the indeterminacymembership is unchanged.

Definition 3. [19] Definition of advantage comparison for interval values. Suppose that there are $\mathrm{n}$ interval numbers $\tilde{a}_{i}=\left[a_{i}^{l}, a_{i}^{r}\right](i=1,2, \ldots, n)$ and that the comparison of each interval number $\tilde{a}_{i}$ to all interval numbers $\tilde{a}_{j}=\left[a_{j}^{l}, a_{j}^{r}\right](j=1,2, \ldots, n)$ is formulated by

$$
p_{i j}\left(\tilde{a}_{i} \geq \tilde{a}_{j}\right)=\max \left\{1-\max \left(\frac{\tilde{a}_{j}^{r}-\tilde{a}_{i}^{l}}{l_{\tilde{a}_{i}}+l_{\tilde{a}_{j}}}, 0\right), 0\right\} .
$$

Then a complementary matrix can be constructed as follows: 


$$
P=\left[\begin{array}{cccc}
p_{11} & p_{12} & \ldots & p_{1 n} \\
p_{21} & p_{22} & \ldots & p_{2 n} \\
\vdots & \\
p_{n 1} & p_{n 2} & \ldots & p_{n n}
\end{array}\right]
$$

where $p_{i j} \geq 0, p_{i j}+p_{j i}=1, p_{i i}=0.5$.

Definition 4. Based on the score function and accuracy function of IVIFSs, the score function, accuracy function and certainty function of an IVNS $A=<$ $\left[T_{A}^{l}, T_{A}^{r}\right],\left[I_{A}^{l}, I_{A}^{r}\right],\left[F_{A}^{l}, F_{A}^{r}\right]>$ are defined as follows.

$$
\text { (1) } \begin{aligned}
s(A)= & {\left[T_{A}^{l}+1-I_{A}^{r}+1-F_{A}^{r},\right.} \\
& \left.T_{A}^{r}+1-I_{A}^{l}+1-F_{A}^{r}\right]
\end{aligned}
$$

(2) $a(A)=\left[\min \left\{T_{A}^{l}-F_{A}^{l}, T_{A}^{r}-F_{A}^{r}\right\}\right.$,

$$
\left.\max \left\{T_{A}^{l}-F_{A}^{l}, T_{A}^{r}-F_{A}^{r}\right\}\right]
$$

$$
\text { (3) } c(A)=\left[T_{A}^{l}, T_{A}^{r}\right]
$$

Definition 5. [4] Let A and B be two IVNSs. The comparison approach can be defined as follows.

If $p(s(A) \geq s(B))>0.5$, then $\mathrm{A}$ is superior to $\mathrm{B}$, denoted by $A \succ B$.

If $p(s(A) \geq s(B))=0.5$, and $p(a(A) \geq a(B))>0.5$, then $\mathrm{A}$ is superior to $\mathrm{B}$, denoted by $A \succ B$.

If $p(s(A) \geq s(B))=0.5, p(a(A) \geq a(B))=0.5$, and $p(c(A) \geq c(B))>0.5$, then $\mathrm{A}$ is superior to $\mathrm{B}$, denoted by $A \succ B$.

If $p(s(A) \geq s(B))=0.5, p(a(A) \geq a(B))=0.5$, and $p(c(A) \geq c(B))=0.5$, then $\mathrm{A}$ is indifferent to $\mathrm{B}$, denoted by $A \sim B$.

Definition 6. Let IVNS $A=<\left[T_{A}^{l}, T_{A}^{r}\right],\left[I_{A}^{l}, I_{A}^{r}\right],\left[F_{A}^{l}\right.$, $\left.F_{A}^{r}\right]>, \quad B=<\left[T_{B}^{l}, T_{B}^{r}\right],\left[I_{B}^{l}, I_{B}^{r}\right],\left[F_{B}^{l}, F_{B}^{r}\right]>$; when $\lambda>0$, the basic operators are defined as follows.

(1) $A+B=<\left[T_{A}^{l}+T_{B}^{l}-T_{A}^{l} T_{B}^{l}, T_{A}^{r}+T_{B}^{r}-T_{A}^{r} T_{B}^{r}\right]$,

$$
\left[I_{A}^{l} I_{B}^{l}, I_{A}^{r} I_{B}^{r}\right],\left[F_{A}^{l} F_{B}^{l}, F_{A}^{r} F_{B}^{r}\right]>
$$

(2) $A \cdot B=<\left[T_{A}^{l} T_{B}^{l}, T_{A}^{r} T_{B}^{r}\right],\left[I_{A}^{l}+I_{B}^{l}-I_{A}^{l} I_{B}^{l}\right.$,

$$
\begin{aligned}
& \left.I_{A}^{r}+I_{B}^{r}-I_{A}^{r} I_{B}^{r}\right],\left[F_{A}^{l}+F_{B}^{l}-F_{A}^{l} F_{B}^{l},\right. \\
& \left.F_{A}^{r}+F_{B}^{r}-F_{A}^{r} F_{B}^{r}\right]>
\end{aligned}
$$

$$
\begin{gathered}
\text { (3) } \begin{aligned}
\lambda A= & <\left[1-\left(1-T_{A}^{l}\right)^{\lambda}, 1-\left(1-T_{A}^{r}\right)^{\lambda}\right], \\
& {\left[\left(I_{A}^{l}\right)^{\lambda},\left(I_{A}^{r}\right)^{\lambda}\right],\left[\left(F_{A}^{l}\right)^{\lambda},\left(F_{A}^{r}\right)^{\lambda}\right]>}
\end{aligned} \text { (4) } A^{\lambda}=<\left[\left(T_{A}^{l}\right)^{\lambda},\left(T_{A}^{r}\right)^{\lambda}\right],\left[1-\left(1-I_{A}^{l}\right)^{\lambda},\right. \\
\left.1-\left(1-I_{A}^{r}\right)^{\lambda}\right],\left[1-\left(1-F_{A}^{l}\right)^{\lambda}, 1-\left(1-F_{A}^{r}\right)^{\lambda}\right]>
\end{gathered}
$$

Theorem 1. Let A, B, C be three IVNSs, $\lambda, \lambda_{1}, \lambda_{2}>0$. The following equations are true.
(1) $A+B=B+A$
(2) $A \cdot B=B \cdot A$
(3) $\lambda(A+B)=\lambda A+\lambda B$
(4) $(A \cdot B)^{\lambda}=A^{\lambda} \cdot B^{\lambda}$
(5) $\lambda_{1} A+\lambda_{2} A=\left(\lambda_{1}+\lambda_{2}\right) A$
(6) $A^{\lambda_{1}} \cdot A^{\lambda_{2}}=A^{\left(\lambda_{1}+\lambda_{2}\right)}$
(7) $(A+B)+C=A+(B+C)$
(8) $(A \cdot B) \cdot C=A \cdot(B \cdot C)$

Proof 1. According to definition 6, (1) and (2) are obvious. The others are proven as follows.

Proof 2. Formula (3):

$$
\begin{aligned}
& \lambda(A+B) \\
& =\lambda\left(<\left[T_{A}^{l}+T_{B}^{l}-T_{A}^{l} T_{B}^{l}, T_{A}^{r}+T_{B}^{r}-T_{A}^{r} T_{B}^{r}\right],\right. \\
& \left.\left[I_{A}^{l} I_{B}^{l}, I_{A}^{r} I_{B}^{r}\right],\left[F_{A}^{l} F_{B}^{l}, F_{A}^{r} F_{B}^{r}\right]>\right) \\
& =<\left[1-\left(1-T_{A}^{l}+T_{B}^{l}+T_{A}^{l} T_{B}^{l}\right)^{\lambda},\right. \\
& \left.1-\left(1-T_{A}^{r}+T_{B}^{r}+T_{A}^{r} T_{B}^{r}\right)^{\lambda}\right], \\
& {\left[\left(I_{A}^{l} I_{B}^{l}\right)^{\lambda},\left(I_{A}^{r} I_{B}^{r}\right)^{\lambda}\right],\left[\left(F_{A}^{l} F_{B}^{l}\right)^{\lambda},\left(F_{A}^{r} F_{B}^{r}\right)^{\lambda}\right]>} \\
& =<\left[1-\left(1-T_{A}^{l}\right)^{\lambda}\left(1-T_{B}^{l}\right)^{\lambda}\right. \text {, } \\
& \left.1-\left(1-T_{A}^{r}\right)^{\lambda}\left(1-T_{B}^{r}\right)^{\lambda}\right], \\
& {\left[\left(I_{A}^{l} I_{B}^{l}\right)^{\lambda},\left(I_{A}^{r} I_{B}^{r}\right)^{\lambda}\right],\left[\left(F_{A}^{l} F_{B}^{l}\right)^{\lambda},\left(F_{A}^{r} F_{B}^{r}\right)^{\lambda}\right]>} \\
& =<\left[1-\left(1-\left(1-T_{A}^{l}\right)^{\lambda}\right)\left(1-\left(1-T_{B}^{l}\right)^{\lambda}\right)\right. \text {, } \\
& \left.1-\left(1-\left(1-T_{A}^{r}\right)^{\lambda}\right)\left(1-\left(1-T_{B}^{r}\right)^{\lambda}\right)\right], \\
& {\left[\left(I_{A}^{l}\right)^{\lambda}\left(I_{B}^{l}\right)^{\lambda},\left(I_{A}^{r}\right)^{\lambda}\left(I_{B}^{r}\right)^{\lambda}\right],} \\
& {\left[\left(F_{A}^{l}\right)^{\lambda}\left(F_{B}^{l}\right)^{\lambda},\left(F_{A}^{r}\right)^{\lambda}\left(F_{B}^{r}\right)^{\lambda}\right]>} \\
& =\left(<\left[1-\left(1-T_{A}^{l}\right)^{\lambda}, 1-\left(1-T_{A}^{r}\right)^{\lambda}\right]\right. \text {, }
\end{aligned}
$$




$$
\begin{aligned}
& {\left.\left[\left(I_{A}^{l}\right) \lambda,\left(I_{A}^{r}\right)^{\lambda}\right],\left[\left(F_{A}^{l}\right)^{\lambda},\left(F_{A}^{r}\right)^{\lambda}\right]>\right) } \\
&+\left(<\left[1-\left(1-T_{B}^{l}\right)^{\lambda}, 1-\left(1-T_{B}^{r}\right)^{\lambda}\right],\right. \\
& {\left.\left[\left(I_{B}^{l}\right) \lambda,\left(I_{B}^{r}\right)^{\lambda}\right],\left[\left(F_{B}^{l}\right)^{\lambda},\left(F_{B}^{r}\right)^{\lambda}\right]>\right) } \\
&=\lambda A+\lambda B
\end{aligned}
$$

Proof 3. Formula (4):

$$
\begin{aligned}
& (A \cdot B)^{\lambda} \\
& =\left(<\left[T_{A}^{l} T_{B}^{l}, T_{A}^{r} T_{B}^{r}\right],\right. \\
& {\left[I_{A}^{l}+I_{B}^{l}-I_{A}^{l} I_{B}^{l}, I_{A}^{r}+I_{B}^{r}-I_{A}^{r} I_{B}^{r}\right],} \\
& \left.\left[F_{A}^{l}+F_{B}^{l}-F_{A}^{l} F_{B}^{l}, F_{A}^{r}+F_{B}^{r}-F_{A}^{r} F_{B}^{r}\right]>\right)^{\lambda} \\
& =<\left[\left(T_{A}^{l} T_{B}^{l}\right)^{\lambda},\left(T_{A}^{r} T_{B}^{r}\right)^{\lambda}\right] \text {, } \\
& {\left[1-\left(1-I_{A}^{l}-I_{B}^{l}+I_{A}^{l} I_{B}^{l}\right)^{\lambda},\right.} \\
& \left.1-\left(1-I_{A}^{r}-I_{B}^{r}+I_{A}^{r} I_{B}^{r}\right)^{\lambda}\right], \\
& {\left[1-\left(1-F_{A}^{l}-F_{B}^{l}+F_{A}^{l} F_{B}^{l}\right)^{\lambda},\right.} \\
& \left.1-\left(1-F_{A}^{r}-F_{B}^{r}+F_{A}^{r} F_{B}^{r}\right)^{\lambda}\right]> \\
& =<\left[\left(T_{A}^{l} T_{B}^{l}\right)^{\lambda},\left(T_{A}^{r} T_{B}^{r}\right)^{\lambda}\right] \text {, } \\
& {\left[1-\left(1-I_{A}^{l}\right)^{\lambda}\left(1-I_{B}^{l}\right)^{\lambda},\right.} \\
& \left.1-\left(1-I_{A}^{r}\right)^{\lambda}\left(1-I_{B}^{r}\right)^{\lambda}\right], \\
& {\left[1-\left(1-F_{A}^{l}\right)^{\lambda}\left(1-F_{B}^{l}\right)^{\lambda},\right.} \\
& \left.1-\left(1-F_{A}^{r}\right)^{\lambda}\left(1-F_{B}^{r}\right)^{\lambda}\right]> \\
& =\left\langle\left[\left(T_{A}^{l}\right)^{\lambda}\left(T_{B}^{l}\right)^{\lambda},\left(T_{A}^{r}\right)^{\lambda}\left(T_{B}^{r}\right)^{\lambda}\right]\right. \\
& {\left[1-\left[1-\left(1-I_{A}^{l}\right)^{\lambda}\right]\left[1-\left(1-I_{B}^{l}\right)^{\lambda}\right],\right.} \\
& \left.1-\left[1-\left(1-I_{A}^{r}\right)^{\lambda}\right]\left[1-\left(1-I_{B}^{r}\right)^{\lambda}\right]\right], \\
& {\left[1-\left[1-\left(1-F_{A}^{l}\right)^{\lambda}\right]\left[1-\left(1-F_{B}^{l}\right)^{\lambda}\right]\right. \text {, }} \\
& \left.\left.1-\left[1-\left(1-F_{A}^{r}\right)^{\lambda}\right]\left[1-\left(1-F_{B}^{r}\right)^{\lambda}\right]\right]\right\rangle \\
& =\left(\left\langle\left[\left(T_{A}^{l}\right)^{\lambda},\left(T_{A}^{r}\right)^{\lambda}\right]\right.\right. \text {, } \\
& {\left[1-\left(1-I_{A}^{l}\right)^{\lambda}, 1-\left(1-I_{A}^{r}\right)^{\lambda}\right],} \\
& \left.\left.\left[1-\left(1-F_{A}^{l}\right)^{\lambda}, 1-\left(1-F_{A}^{r}\right)^{\lambda}\right]\right\rangle\right)
\end{aligned}
$$

Proof 4. Formula (5):

$$
\begin{aligned}
\lambda_{1} A+ & \lambda_{2} A \\
= & \left\langle\left[1-\left(1-T_{A}^{l}\right)^{\lambda_{1}}, 1-\left(1-T_{A}^{r}\right)^{\lambda_{1}}\right],\right. \\
& {\left.\left.\left[\left(I_{A}^{l}\right)^{\lambda_{1}},\left(I_{A}^{r}\right)^{\lambda_{1}}\right],\left[\left(F_{A}^{l}\right)^{\lambda_{1}},\left(F_{A}^{r}\right)^{\lambda_{1}}\right]\right\rangle\right) } \\
+ & \left(\left\langle\left[1-\left(1-T_{A}^{l}\right)^{\lambda_{2}}, 1-\left(1-T_{A}^{r}\right)^{\lambda_{2}}\right],\right.\right. \\
& {\left.\left.\left[\left(I_{A}^{l}\right)^{\lambda_{2}},\left(I_{A}^{r}\right)^{\lambda_{2}}\right],\left[\left(F_{A}^{l}\right)^{\lambda_{2}},\left(F_{A}^{r}\right)^{\lambda_{2}}\right]\right\rangle\right) } \\
=\langle & {\left[1-\left[1-\left(1-T_{A}^{l}\right)^{\lambda_{1}}\right]\left[1-\left(1-T_{A}^{l}\right)^{\lambda_{2}}\right],\right.} \\
& \left.1-\left[1-\left(1-T_{A}^{r}\right)^{\lambda_{1}}\right]\left[1-\left(1-T_{A}^{r}\right)^{\lambda_{2}}\right]\right], \\
& {\left[\left(I_{A}^{l}\right)^{\lambda_{1}}\left(I_{A}^{l}\right)^{\lambda_{2}},\left(I_{A}^{r}\right)^{\lambda_{1}}\left(I_{A}^{r}\right)^{\lambda_{2}}\right], } \\
& {\left.\left[\left(F_{A}^{l}\right)^{\lambda_{1}}\left(F_{A}^{l}\right)^{\lambda_{2}},\left(F_{A}^{r}\right)^{\lambda_{1}}\left(F_{A}^{r}\right)^{\lambda_{2}}\right]\right\rangle } \\
=\langle & {\left[1-\left(1-T_{A}^{l}\right)^{\lambda_{1}}\left(1-T_{A}^{l}\right)^{\lambda_{2}},\right.} \\
& \left.1-\left(1-T_{A}^{r}\right)^{\lambda_{1}}\left(1-T_{A}^{r}\right)^{\lambda_{2}}\right], \\
=\left(\lambda_{1}+\lambda_{2}\right) A & {\left[\left(I_{A}^{l}\right)^{\lambda_{1}}\left(I_{A}^{l}\right)^{\lambda_{2}},\left(I_{A}^{r}\right)^{\lambda_{1}}\left(I_{A}^{r}\right)^{\lambda_{2}}\right], } \\
=\langle & {\left.\left[\left(F_{A}^{l}\right)^{\lambda_{1}}\left(F_{A}^{l}\right)^{\lambda_{2}},\left(F_{A}^{r}\right)^{\lambda_{1}}\left(F_{A}^{r}\right)^{\lambda_{2}}\right]\right\rangle } \\
& {\left[\left(I_{A}^{l}\right)^{\left(\lambda_{1}+\lambda_{2}\right)},\left(I_{A}^{r}\right)^{\left(\lambda_{1}+\lambda_{2}\right)}\right], } \\
& {\left.\left[\left(F_{A}^{l}\right)^{\left(\lambda_{1}+\lambda_{2}\right)},\left(F_{A}^{r}\right)^{\left(\lambda_{1}+\lambda_{2}\right)}\right]\right\rangle } \\
l & \left(1-T_{A}^{r}\right)^{\left(\lambda_{1}+\lambda_{2}\right)}, \\
\left.\lambda_{1}\right) & \\
= &
\end{aligned}
$$


Proof 5. Formula (6):

$$
\begin{aligned}
& A^{\lambda_{1}} \cdot A^{\lambda_{2}}=\langle \\
&=\langle\left.\left(T_{A}^{l}\right)^{\lambda_{1}},\left(T_{A}^{r}\right)^{\lambda_{1}}\right],\left[1-\left(1-I_{A}^{l}\right)^{\lambda_{1}}, 1-\left(1-I_{A}^{r}\right)^{\lambda_{1}}\right], \\
& {\left.\left.\left[1-\left(1-F_{A}^{l}\right)^{\lambda_{1}}, 1-\left(1-F_{A}^{r}\right)^{\lambda_{1}}\right]\right\rangle\right) \cdot\left(\left\langle\left[\left(T_{A}^{l}\right)^{\lambda_{2}},\left(T_{A}^{r}\right)^{\lambda_{2}}\right],\right.\right.} \\
& {\left.\left.\left[1-\left(1-I_{A}^{l}\right)^{\lambda_{2}}, 1-\left(1-I_{A}^{r}\right)^{\lambda_{2}}\right],\left[1-\left(1-F_{A}^{l}\right)^{\lambda_{2}}, 1-\left(1-F_{A}^{r}\right)^{\lambda_{2}}\right]\right\rangle\right) } \\
&=\langle {\left[\left(T_{A}^{l}\right)^{\lambda_{1}}\left(T_{A}^{l}\right)^{\lambda_{2}},\left(T_{A}^{r}\right)^{\lambda_{1}}\left(T_{A}^{r}\right)^{\lambda_{2}}\right],\left[1-\left[1-\left(1-I_{A}^{l}\right)^{\lambda_{1}}\right]\left[1-\left(1-I_{A}^{l}\right)^{\lambda_{2}}\right],\right.} \\
&\left.1-\left[1-\left(1-I_{A}^{r}\right)^{\lambda_{1}}\right]\left[1-\left(1-I_{A}^{r}\right)^{\lambda_{2}}\right]\right] \\
&\left\langle\left[1-\left[1-\left(1-I_{A}^{l}\right)^{\lambda_{1}}\right]\left[1-\left(1-I_{A}^{l}\right)^{\lambda_{2}}\right], 1-\left[1-\left(1-I_{A}^{r}\right)^{\lambda_{1}}\right]\left[1-\left(1-I_{A}^{r}\right)^{\lambda_{2}}\right]\right],\right. \\
& {\left.\left[1-\left[1-\left(1-F_{A}^{l}\right)^{\lambda_{1}}\right]\left[1-\left(1-F_{A}^{l}\right)^{\lambda_{2}}\right], 1-\left[1-\left(1-F_{A}^{r}\right)^{\lambda_{1}}\right]\left[1-\left(1-F_{A}^{r}\right)^{\lambda_{2}}\right]\right]\right\rangle } \\
&=\langle {\left[\left(T_{A}^{l}\right)^{\lambda_{1}}\left(T_{A}^{l}\right)^{\lambda_{2}},\left(T_{A}^{r}\right)^{\lambda_{1}}\left(T_{A}^{r}\right)^{\lambda_{2}}\right],\left[1-\left(1-I_{A}^{l}\right)^{\lambda_{1}}\left(1-I_{A}^{l}\right)^{\lambda_{2}}, 1-\left(1-I_{A}^{r}\right)^{\lambda_{1}}\left(1-I_{A}^{r}\right)^{\lambda_{2}}\right], } \\
& {\left.\left[1-\left(1-F_{A}^{l}\right)^{\lambda_{1}}\left(1-F_{A}^{l}\right)^{\lambda_{2}}, 1-\left(1-F_{A}^{r}\right)^{\lambda_{1}}\left(1-F_{A}^{r}\right)^{\lambda_{2}}\right]\right\rangle } \\
&=\langle {\left[\left(T_{A}^{l}\right)^{\left(\lambda_{1}+\lambda_{2}\right)},\left(T_{A}^{r}\right)^{\left(\lambda_{1}+\lambda_{2}\right)}\right],\left[1-\left(1-I_{A}^{l}\right)^{\left(\lambda_{1}+\lambda_{2}\right)}, 1-\left(1-I_{A}^{r}\right)^{\left(\lambda_{1}+\lambda_{2}\right)}\right], } \\
& {\left.\left[1-\left(1-F_{A}^{l}\right)^{\left(\lambda_{1}+\lambda_{2}\right)}, 1-\left(1-F_{A}^{r}\right)^{\left(\lambda_{1}+\lambda_{2}\right)}\right]\right\rangle } \\
&=A^{\left(\lambda_{1}+\lambda_{2}\right)}
\end{aligned}
$$

Proof 6. Formula (7):

$$
\begin{aligned}
(A+B)+C=(\langle & {\left[T_{A}^{l}+T_{B}^{l}-T_{A}^{l} T_{B}^{l}, T_{A}^{r}+T_{B}^{r}-T_{A}^{r} T_{B}^{r}\right],\left[I_{A}^{l} I_{B}^{l}, I_{A}^{r} I_{B}^{r}\right], } \\
& \left.\left.\quad\left[F_{A}^{l} F_{B}^{l}, F_{A}^{r} F_{B}^{r}\right]\right\rangle\right)+C \\
= & \left(\left\langle\left[1-\left(1-T_{A z a}^{l}\right)\left(1-T_{B}^{l}\right)\right],\left[I_{A}^{l} I_{B}^{l}, I_{A}^{r} I_{B}^{r}\right],\left[F_{A}^{l} F_{B}^{l}, F_{A}^{r} F_{B}^{r}\right]\right\rangle\right)+C \\
= & \left\langle\left[1-\left\{1-\left[1-\left(1-T_{A}^{l}\right)\left(1-T_{B}^{l}\right)\right]\right\}\left(1-T_{C}^{l}\right)\right],\left[I_{A}^{l} I_{B}^{l} I_{C}^{l}, I_{A}^{r} I_{B}^{r} I_{C}^{r}\right],\right. \\
= & \left.\quad\left[F_{A}^{l} F_{B}^{l} F_{C}^{l}, F_{A}^{r} F_{B}^{r} F_{C}^{r}\right]\right\rangle \\
= & \left.\left\langle 1-\left(1-T_{A}^{l}\right)\left(1-T_{B}^{l}\right)\left(1-T_{C}^{l}\right)\right],\left[I_{A}^{l} I_{B}^{l} I_{C}^{l}, I_{A}^{r} I_{B}^{r} I_{C}^{r}\right],\left[F_{A}^{l} F_{B}^{l} F_{C}^{l}, F_{A}^{r} F_{B}^{r} F_{C}^{r}\right]\right\rangle \\
= & \left\langle\left[1-\left(1-T_{A}^{l}\right)\left\{1-\left[1-\left(1-T_{B}^{l}\right)\left(1-T_{C}^{l}\right)\right]\right\}\right],\left[I_{A}^{l} I_{B}^{l} I_{C}^{l}, I_{A}^{r} I_{B}^{r} I_{C}^{r}\right],\right. \\
& \left.\quad\left[F_{A}^{l} F_{B}^{l} F_{C}^{l}, F_{A}^{r} F_{B}^{r} F_{C}^{r}\right]\right\rangle \\
= & A+\left(\left\langle\left[1-\left(1-T_{B}^{l}\right)\left(1-T_{C}^{l}\right)\right],\left[I_{B}^{l} I_{C}^{l}, I_{B}^{r} I_{C}^{r}\right],\left[F_{B}^{l} F_{C}^{l}, F_{B}^{r} F_{C}^{r}\right]\right\rangle\right) \\
= & A+\left(\left\langle\left[T_{B}^{l}+T_{C}^{l}-T_{B}^{l} T_{C}^{l}, T_{B}^{r}+T_{C}^{r}-T_{B}^{r} T_{C}^{r}\right],\left[I_{B}^{l} I_{C}^{l}, I_{B}^{r} I_{C}^{r}\right],\left[F_{B}^{l} F_{C}^{l}, F_{B}^{r} F_{C}^{r}\right]\right\rangle\right) \\
= & A+(B+C)
\end{aligned}
$$


Proof 7. Formula (8):

$(A \cdot B) \cdot C$

$$
\begin{aligned}
& =\left(\left\langle\left[T_{A}^{l} T_{B}^{l}, T_{A}^{r} T_{B}^{r}\right],\left[I_{A}^{l}+I_{B}^{l}-I_{A}^{l} I_{B}^{l}, I_{A}^{r}+I_{B}^{r}-I_{A}^{r} I_{B}^{r}\right], \quad\left[F_{A}^{l}+F_{B}^{l}-F_{A}^{l} F_{B}^{l}, F_{A}^{r}+F_{B}^{r}-F_{A}^{r} F_{B}^{r}\right]\right\rangle\right) \cdot C \\
& =\left(\left\langle\left[T_{A}^{l} T_{B}^{l}, T_{A}^{r} T_{B}^{r}\right],\left[1-\left(1-I_{A}^{l}\right)\left(1-I_{B}^{l}\right)\right],\left[1-\left(1-F_{A}^{l}\right)\left(1-F_{B}^{l}\right)\right]\right\rangle\right) \cdot C \\
& =\left\langle\left[T_{A}^{l} T_{B}^{l} T_{C}^{l}, T_{A}^{r} T_{B}^{r} T_{C}^{r}\right],\left[1-\left\{1-\left[1-\left(1-I_{A}^{l}\right)\left(1-I_{B}^{l}\right)\right]\right\}\left(1-I_{C}^{l}\right)\right],\left[1-\left\{1-\left[1-\left(1-F_{A}^{l}\right)\left(1-F_{B}^{l}\right)\right]\right\}\left(1-F_{C}^{l}\right)\right]\right\rangle \\
& =\left\langle\left[T_{A}^{l} T_{B}^{l} T_{C}^{l}, T_{A}^{r} T_{B}^{r} T_{C}^{r}\right],\left[1-\left(1-I_{A}^{l}\right)\left(1-I_{B}^{l}\right)\left(1-I_{C}^{l}\right)\right],\left[1-\left(1-F_{A}^{l}\right)\left(1-F_{B}^{l}\right)\left(1-F_{C}^{l}\right)\right]\right\rangle \\
& =\left\langle\left[T_{A}^{l} T_{B}^{l} T_{C}^{l}, T_{A}^{r} T_{B}^{r} T_{C}^{r}\right],\left[1-\left(1-I_{A}^{l}\right)\left\{1-\left[1-\left(1-I_{B}^{l}\right)\left(1-I_{C}^{l}\right)\right]\right\}\right],\left[1-\left(1-F_{A}^{l}\right)\left\{1-\left[1-\left(1-F_{B}^{l}\right)\left(1-F_{C}^{l}\right)\right]\right\}\right]\right\rangle \\
& =A \cdot\left(\left\langle\left[T_{B}^{l} T_{C}^{l}, T_{B}^{r} T_{C}^{r}\right],\left[1-\left(1-I_{B}^{l}\right)\left(1-I_{C}^{l}\right)\right],\left[1-\left(1-F_{B}^{l}\right)\left(1-F_{C}^{l}\right)\right]\right\rangle\right) \\
& =A \cdot\left(\left\langle\left[T_{B}^{l} T_{C}^{l}, T_{B}^{r} T_{C}^{r}\right],\left[I_{B}^{l}+I_{C}^{l}-I_{B}^{l} I_{C}^{l}, I_{B}^{r}+I_{C}^{r}-I_{B}^{r} I_{C}^{r}\right],\left[F_{B}^{l}+F_{C}^{l}-F_{B}^{l} F_{C}^{l}, F_{B}^{r}+F_{C}^{r}-F_{B}^{r} F_{C}^{r}\right]\right\rangle\right)=A \cdot(B \cdot C)
\end{aligned}
$$

Definition 7. Let $A_{j}=<\left[T_{A_{j}}^{l}, T_{A_{j}}^{r}\right],\left[I_{A_{j}}^{l}, I_{A_{j}}^{r}\right],\left[F_{A_{j}}^{l}\right.$, $F_{A_{j}}^{r}$ ] > be a collection of IVNSs, and $W=\left(w_{1}, w_{2}\right.$, $\left.\ldots, w_{n}\right)^{T}$ be the weight vector of $A_{j}$, with $w_{j} \geq 0$, and $\sum_{j=1}^{n} w_{j}=1$. Their generalized weighted aggregation GWA [16] $A^{n} \rightarrow A$ is defined as follows.

$$
\begin{aligned}
& Z=\operatorname{IVNSGWA}\left(A_{1}, A_{2}, \ldots, A_{n}\right)=\left(\sum_{j=1}^{n} w_{j} A_{j}^{\lambda}\right)^{1 / \lambda} \\
& =\left(\sum_{j=1}^{n} w_{j}\left(\left\langle\left[\left(T_{j}^{l}\right)^{\lambda},\left(T_{j}^{r}\right)^{\lambda}\right],\left[1-\left(1-I_{j}^{l}\right)^{\lambda}, 1-\left(1-I_{j}^{r}\right)^{\lambda}\right],\left[1-\left(1-F_{j}^{l}\right)^{\lambda}, 1-\left(1-F_{j}^{r}\right)^{\lambda}\right]\right\rangle\right)\right)^{1 / \lambda} \\
& =\left(\sum _ { j = 1 } ^ { n } w _ { j } \left(\left\langle\left[1-\left(1-\left(T_{j}^{l}\right)^{\lambda}\right)^{w_{j}}, 1-\left(1-\left(T_{j}^{r}\right)^{\lambda}\right)^{w_{j}}\right],\left[\left(1-\left(1-I_{j}^{l}\right)^{\lambda}\right)^{w_{j}},\left(1-\left(1-I_{j}^{r}\right)^{\lambda}\right)^{w_{j}}\right],\right.\right.\right. \\
& \left.\left.\left.\left[\left(1-\left(1-F_{j}^{l}\right)^{\lambda}\right)^{w_{j}},\left(1-\left(1-F_{j}^{r}\right)^{\lambda}\right)^{w_{j}}\right]\right\rangle\right)\right)^{1 / \lambda} \\
& =\left(\left\langle\left[1-\prod_{j=1}^{n}\left(1-\left(T_{j}^{l}\right)^{\lambda}\right)^{w_{j}}, 1-\prod_{j=1}^{n}\left(1-\left(T_{j}^{r}\right)^{\lambda}\right)^{w_{j}}\right],\left[\prod_{j=1}^{n}\left(1-\left(1-I_{j}^{l}\right)^{\lambda}\right)^{w_{j}}, \prod_{j=1}^{n}\left(1-\left(1-I_{j}^{r}\right)^{\lambda}\right)^{w_{j}},\right]\right.\right. \text {, } \\
& \left.\left.\left[\prod_{j=1}^{n}\left(1-\left(1-I_{j}^{l}\right)^{\lambda}\right)^{w_{j}}, \prod_{j=1}^{n}\left(1-\left(1-I_{j}^{r}\right)^{\lambda}\right)^{w_{j}},\right]\right\rangle\right)^{1 / \lambda} \\
& =\left(\left\langle\left[\left(1-\prod_{j=1}^{n}\left(1-\left(T_{j}^{l}\right)^{\lambda}\right)^{w_{j}}\right)^{1 / \lambda},\left(1-\prod_{j=1}^{n}\left(1-\left(T_{j}^{r}\right)^{\lambda}\right)^{w_{j}}\right)^{1 / \lambda}\right],\right.\right. \\
& {\left[1-\left(1-\prod_{j=1}^{n}\left(1-\left(1-I_{j}^{l}\right)^{\lambda}\right)^{w_{j}}\right)^{1 / \lambda}, 1-\left(1-\prod_{j=1}^{n}\left(1-\left(1-I_{j}^{r}\right)^{\lambda}\right)^{w_{j}}\right)^{1 / \lambda}\right],} \\
& \left.\left.\left[1-\left(1-\prod_{j=1}^{n}\left(1-\left(1-F_{j}^{l}\right)^{\lambda}\right)^{w_{j}}\right)^{1 / \lambda}, 1-\left(1-\prod_{j=1}^{n}\left(1-\left(1-F_{j}^{r}\right)^{\lambda}\right)^{w_{j}}\right)^{1 / \lambda}\right]\right\rangle\right)
\end{aligned}
$$


When $\lambda \rightarrow 0$

$$
\begin{aligned}
Z= & \operatorname{IVNSGWA}\left(A_{1}, A_{2}, \ldots, A_{n}\right)=\prod_{j=1}^{n} A_{j}^{w_{j}} \\
= & \prod_{j=1}^{n}\left(\left\langle\left[\left(T_{j}^{l}\right)^{w_{j}},\left(T_{j}^{r}\right)^{w_{j}}\right],\right.\right. \\
& {\left[1-\left(1-I_{j}^{l}\right)^{w_{j}}, 1-\left(1-I_{j}^{r}\right)^{w_{j}}\right], } \\
& {\left.\left.\left[1-\left(1-F_{j}^{l}\right)^{w_{j}}, 1-\left(1-F_{j}^{r}\right)^{w_{j}}\right]\right\rangle\right) } \\
= & \left(\left\langle\left[\prod_{j=1}^{n}\left(T_{j}^{l}\right)^{w_{j}}, \prod_{j=1}^{n}\left(T_{j}^{r}\right)^{w_{j}}\right],\right.\right. \\
& {\left[1-\prod_{j=1}^{n}\left(1-I_{j}^{l}\right)^{w_{j}}, 1-\prod_{j=1}^{n}\left(1-I_{j}^{r}\right)^{w_{j}}\right], } \\
& {\left.\left.\left[1-\prod_{j=1}^{n}\left(1-F_{j}^{l}\right)^{w_{j}}, 1-\prod_{j=1}^{n}\left(1-F_{j}^{r}\right)^{w_{j}}\right]\right)\right\rangle }
\end{aligned}
$$

When $\lambda=1$

$$
\begin{aligned}
& Z= \operatorname{IVNSGWA}\left(A_{1}, A_{2}, \ldots, A_{n}\right)=\sum_{j=1}^{n} w_{j} A_{j} \\
&=\sum_{j=1}^{n}\left(\left\langle\left[1-\left(1-T_{j}^{l}\right)^{w_{j}}, 1-\left(1-T_{j}^{r}\right)^{w_{j}}\right],\right.\right. \\
&\left.=\left(\left\langle\left[I_{j}^{l}\right)^{w_{j}},\left(I_{j}^{r}\right)^{w_{j}}\right],\left[\left(F_{j}^{l}\right)^{w_{j}},\left(F_{j}^{r}\right)^{w_{j}}\right]\right)\right\rangle \\
&\left.=\prod_{j=1}^{n}\left(1-T_{j}^{l}\right)^{w_{j}}, 1-\prod_{j=1}^{n}\left(1-T_{j}^{r}\right)^{w_{j}}\right] \\
& {\left[\prod_{j=1}^{n}\left(I_{j}^{l}\right)^{w_{j}}, \prod_{j=1}^{n}\left(I_{j}^{r}\right)^{w_{j}}\right], } \\
& {\left.\left.\left[\prod_{j=1}^{n}\left(F_{j}^{l}\right)^{w_{j}}, \prod_{j=1}^{n}\left(F_{j}^{r}\right)^{w_{j}}\right]\right\rangle\right) }
\end{aligned}
$$

When $\lambda=1$, Formula (19) is reduced to Formula (21).

\section{Multi-attribute decision-making method based on IVNSGWA operators}

Definition 7 indicates that:
(1) When $\lambda \rightarrow 0, \operatorname{IVNSGWA}\left(A_{1}, A_{2}, \ldots, A_{n}\right)=$ $\prod_{j=1}^{n} A_{j}^{w_{j}}$, the standard aggregation operator IVNSGWA is reduced to weighted geometric aggregation operator (WG).

(2) When $\lambda=1, \operatorname{IVNSGWA}\left(A_{1}, A_{2}, \ldots, A_{n}\right)=$ $\sum_{j=1}^{n} w_{j} A_{j}$, the standard aggregation operator IVNSGWA is reduced to weighted arithmetic aggregation operator (WA).

(3) Let

$$
\mathrm{Z}=\operatorname{IVNSGWA}\left(A_{1}, A_{2}, \ldots, A_{n}\right) \text {. }
$$

When $Z=\left(\sum_{j=1}^{n} w_{j} A_{j}^{\lambda}\right)^{1 / \lambda}$, the value of $\sum_{j=1}^{n} w_{j}\left(Z-A_{j}^{\lambda}\right)^{2}$ is at its minimum. Therefore, the aggregation operator IVNSGWA is the greatest approximation representing $\mathrm{n}$ attributes.

A multi-attribute decision-making problem represents a process by which to find the best alternative from all of feasible alternatives, in which all alternatives can be evaluated according to a number of attributes or criteria. Let $A=\left\{A_{1}, A_{2}, \ldots, A_{m}\right\}$ be a set of alternatives and $C=\left\{C_{1}, C_{2}, \ldots, C_{n}\right\}$ be a set of attributes. Assume that the vector of the weight $w=\left(w_{1}, w_{2}, \ldots, w_{n}\right)$, where $w_{j} \in[0,1]$, and $\sum_{j=1}^{n} w_{j}=1$. In this case, the characteristic of attribute $C_{j}$ of alternative $A_{i}$ is represented by an IVNS $r_{i j}=\left\langle\left[T_{r_{i j}}^{l}, T_{r_{i j}}^{r}\right],\left[I_{r_{i j}}^{l}, I_{r_{i j}}^{r}\right],\left[F_{r_{i j}}^{l}, F_{r_{i j}}^{r}\right]\right\rangle$. Here, $\left[T_{r_{i j}}^{l}, T_{r_{i j}}^{r}\right],\left[I_{r_{i j}}^{l}, I_{r_{i j}}^{r}\right]$, and $\left[F_{r_{i j}}^{l}, F_{r_{i j}}^{r}\right]$ respectively indicate the degree to which alternative $A_{i}$ satisfies the attribute $C_{j}$, the indeterminacy degree to which the alternative $A_{i}$ satisfies or does not satisfy the attribute $C_{j}$, and the degree to which the alternative $A_{i}$ does not satisfy the attribute $C_{j}$. Therefore, an interval valued neutrosophic decision matrix can be elicited: $R=$ $\left[r_{i j}\right]_{m \times n}$.

The steps of interval valued neutrosophic multiattribute decision-making based on IVNSGWA can be presented as follows.

Step 1. Construction of standard decision matrix.

If $C_{j}$ is a benefit attribute, $b_{i j}=\left\langle\left[T_{r_{i j}}^{l}, T_{r_{i j}}^{r}\right]\right.$, $\left.\left[I_{r_{i j}}^{l}, I_{r_{i j}}^{r}\right],\left[F_{r_{i j}}^{l}, F_{r_{i j}}^{r}\right]\right\rangle$.

If $C_{j}$ is a cost attribute, $b_{i j}=\left\langle\left[F_{r_{i j}}^{l}, F_{r_{i j}}^{r}\right]\right.$, $\left.\left[I_{r_{i j}}^{r}, I_{r_{i j}}^{l}\right],\left[T_{r_{i j}}^{l}, T_{r_{i j}}^{r}\right]\right\rangle$.

Thus, a standard decision matrix is obtained, $B=$ $\left[b_{i j}\right]_{m \times n}$.

Step 2. Calculation of the aggregation value $Z_{i}$ for alternative $a_{i}$. See Definition 8 .

Step 3. Construction of dominance matrices.

Based on Definition 4, calculate score $s\left(Z_{i}\right)$, degree of accuracy $a\left(Z_{i}\right)$ and degree of certainty $c\left(Z_{i}\right)$ for alternative $a_{i}$. Then, according to Definition 3 , construct the 
score dominance matrix $P_{S}$, accuracy dominance matrix $P_{a}$ and certainty dominance matrix $P_{c}$ as follows.

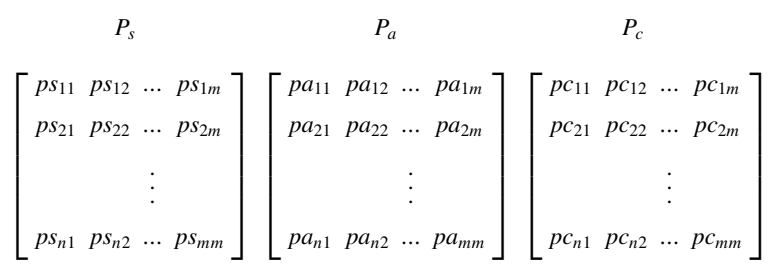

Step 4. Calculation of the total score $p s_{i}$, total accuracy $p a_{i}$ and total certainty $p c_{i}$ for alternative $a_{i}$.

$$
p s_{i}=\sum_{j=1}^{m} p s_{i j} \quad p a_{i}=\sum_{j=1}^{m} p a_{i j} \quad p c_{i}=\sum_{j=1}^{m} p c_{i j}
$$

Step 5. According to Definition 5, determine the ranking order for each alternative.

\section{Illustrative example}

In this section, an example of the multi-attribute decision-making problem of alternatives is used to demonstrate the application and effectiveness of the proposed decision-making method.

Let us consider the decision-making problem adapted from Chi and Liu, in which an investment company wants to invest in the best option [15].

There are four possible alternatives in which to invest, expressed as $\left\{A_{1}, A_{2}, A_{3}, A_{4}\right\}$, where $A_{1}$ is a bookshop, $A_{2}$ is a chemical plant, $A_{3}$ is a supermarket, and $A_{4}$ is a food company.

The investment company must make a decision according to the following three attributes: $C_{1}$ is the earning estimate analysis, $C_{2}$ is the growth analysis, and $C_{3}$ is the environmental impact analysis for the alternatives; $C_{1}$ and $C_{2}$ are benefit attributes, while $C_{3}$ is a cost attribute. The weight vector of the attribute is given by $w=(0.2,0.25,0.55)$. The four possible alternatives are evaluated according to the above three attributes by IVNSs, as shown in the following interval valued neutrosophic decision matrix $\mathrm{R}$.

$$
R=\begin{aligned}
&\langle\langle[0.4,0.5],[0.2,0.3],[0.3,0.4]\rangle \\
&\langle[0.6,0.7],[0.1,0.2],[0.2,0.3]\rangle \\
&\langle[0.3,0.6],[0.2,0.3],[0.3,0.4]\rangle \\
&\langle[0.7,0.8],[0.0,0.1],[0.1,0.2]\rangle \\
&\langle[0.4,0.6],[0.1,0.3],[0.2,0.4]\rangle \\
&\langle[0.6,0.7],[0.1,0.2],[0.2,0.3]\rangle \\
&\langle[0.5,0.6],[0.2,0.3],[0.3,0.4]\rangle \\
&\langle[0.6,0.7],[0.1,0.2],[0.1,0.3]\rangle \\
&\langle[0.7,0.9],[0.2,0.3],[0.4,0.5]\rangle \\
&\langle[0.3,0.6],[0.3,0.5],[0.8,0.9]\rangle \\
&\langle[0.4,0.5],[0.2,0.4],[0.7,0.9]\rangle \\
&\langle[0.6,0.7],[0.3,0.4],[0.8,0.9]\rangle]
\end{aligned}
$$

Each IVNS value in the matrix represents the assessment of the corresponding attribute of a certain alternative. For instance, the first value $<[0.4,0.5],[0.2,0.3],[0.3,0.4]>$ in matrix $\mathrm{R}$ represents the possibility of earning of alternative $A_{1}$, which lies between 0.4 and 0.5 ; the uncertainty of earning of alternative $A_{1}$, which lies between 0.2 and 0.3 ; and the possibility of loss of alternative $A_{1}$, which lies between 0.3 and 0.4 .

(1) Construction of standard decision matrix B.

$$
B=\begin{aligned}
&\langle\langle[0.4,0.5],[0.2,0.3],[0.3,0.4]\rangle \\
&\langle[0.6,0.7],[0.1,0.2],[0.2,0.3]\rangle \\
&\langle[0.3,0.6],[0.2,0.3],[0.3,0.4]\rangle \\
&\langle[0.7,0.8],[0.0,0.1],[0.1,0.2]\rangle \\
&\langle[0.4,0.6],[0.1,0.3],[0.2,0.4]\rangle \\
&\langle[0.6,0.7],[0.1,0.2],[0.2,0.3]\rangle \\
&\langle[0.5,0.6],[0.2,0.3],[0.3,0.4]\rangle \\
&\langle[0.6,0.7],[0.1,0.2],[0.1,0.3]\rangle \\
&\langle[0.4,0.5],[0.2,0.3],[0.7,0.9]\rangle \\
&\langle[0.8,0.9],[0.3,0.5],[0.3,0.6]\rangle \\
&\langle[0.7,0.9],[0.2,0.4],[0.4,0.5]\rangle \\
&\langle[0.8,0.9],[0.3,0.4],[0.6,0.7]\rangle]
\end{aligned}
$$

Since $C_{3}$ is a cost attribute, it is transformed into a benefit attribute according to Definition 2 .

(2) Calculation of aggregation value $Z_{i}$ for alternative $a_{i}$.

According to formula (22), let $\lambda=1$. Thus: 
Table 1

Ranking orders with various $\lambda$

\begin{tabular}{lll}
\hline$\lambda$ & Total score $p s$ & Ranking order \\
\hline$\rightarrow 0$ & {$[0.8609,2.7000,1.5887,2.8504]$} & $A_{4} \succ A_{2} \succ A_{3} \succ A_{1}$ \\
0.1 & {$[0.9307,2.5811,1.4059,3.0823]$} & $A_{4} \succ A_{2} \succ A_{3} \succ A_{1}$ \\
0.3 & {$[0.9347,2.5790,1.3995,3.0868]$} & $A_{4} \succ A_{2} \succ A_{3} \succ A_{1}$ \\
0.5 & {$[0.9385,2.5768,1.3934,3.0913]$} & $A_{4} \succ A_{2} \succ A_{3} \succ A_{1}$ \\
1.0 & {$[0.9467,2.5721,1.3793,3.1019]$} & $A_{4} \succ A_{2} \succ A_{3} \succ A_{1}$ \\
1.4 & {$[0.9516,2.5690,1.3694,3.1100]$} & $A_{4} \succ A_{2} \succ A_{3} \succ A_{1}$ \\
1.8 & {$[0.9549,2.5665,1.3611,3.1175]$} & $A_{4} \succ A_{2} \succ A_{3} \succ A_{1}$ \\
2.0 & {$[0.9560,2.5655,1.3575,3.1210]$} & $A_{4} \succ A_{2} \succ A_{3} \succ A_{1}$ \\
5.0 & {$[0.9425,2.5644,1.3362,3.1569]$} & $A_{4} \succ A_{2} \succ A_{3} \succ A_{1}$ \\
10 & {$[0.8902,2.5815,1.3446,3.1837]$} & $A_{4} \succ A_{2} \succ A_{3} \succ A_{1}$ \\
\hline
\end{tabular}

$$
\begin{aligned}
& Z \\
& =\left[\begin{array}{c}
\langle[0.4000,0.5577],[0.1366,0.3000],[0.2967,0.4899]\rangle \\
\langle[0.6636,0.7720],[0.1316,0.2515],[0.2213,0.3568]\rangle \\
\langle[0.5293,0.7172],[0.2000,0.3224],[0.3224,0.4229]\rangle \\
\langle[0.6824,0.7898],[0.0000,0.2071],[0.1565,0.3419]\rangle
\end{array}\right]
\end{aligned}
$$

(3) Construction of dominance matrices.

According to Definition 4, the score, degree of accuracy and degree of certainty of each alternative are expressed as follows.

$$
\begin{aligned}
& S_{-} A_{-} C(Z) \\
& =\left[\begin{array}{l}
{[1.6101,2.1245][0.0679,0.1033][0.4000,0.5577]} \\
{[2.0554,2.4191][0.4153,0.4423][0.6636,0.7720]} \\
{[1.7840,2.1948][0.2069,0.2942][0.5293,0.7172]} \\
{[2.1335,2.6333][0.4479,0.5259][0.6824,0.7898]}
\end{array}\right]
\end{aligned}
$$

Next, according to Definition 3, the respective dominance matrices are obtained.

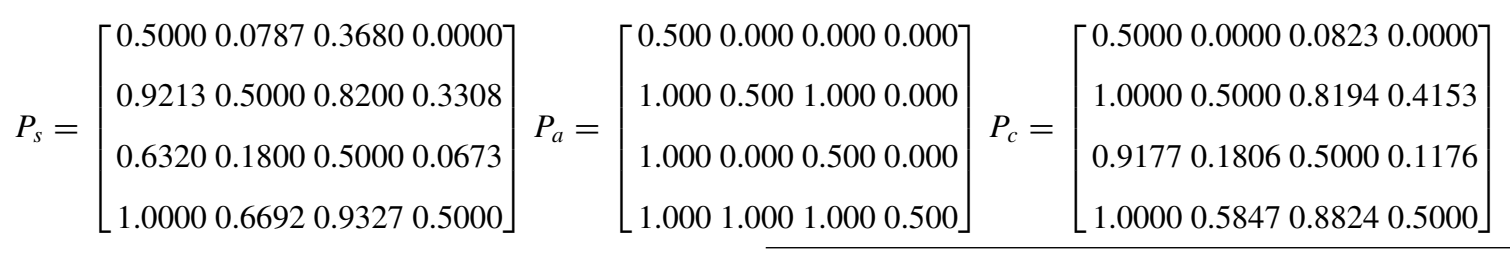

(4) Calculation of the total score $p s_{i}$, total accuracy $p a_{i}$ and total certainty $p c_{i}$ for each alternative.

$$
\begin{aligned}
p_{s} & =\left[\begin{array}{llll}
0.9467 & 2.5721 & 1.3793 & 3.1019
\end{array}\right] \\
p_{a} & =\left[\begin{array}{llll}
0.5000 & 2.5000 & 1.5000 & 3.5000
\end{array}\right] \\
p_{c} & =\left[\begin{array}{llll}
0.5823 & 2.7347 & 1.7159 & 2.9671
\end{array}\right]
\end{aligned}
$$

(5) Ranking the alternatives.

The scores of the three alternatives vary, so the ranking order is $A_{4} \succ A_{2} \succ A_{3} \succ A_{1}$ according to $p s$, which is identical to the ranking order used in the [15] TOPSIS method.

In order to discuss the impact of parameter $\lambda$, the ranking order under different $\lambda$ were calculated. The ranking orders are listed in Table 1 .

As indicated by Table 1, the total score of each alternative varies for different values of parameter $\lambda$ while the ranking order remains unchanged. In fact, $\lambda$ generally represents the attitude of decision makers; smaller $\lambda$ indicates more conservative attitudes, while larger $\lambda$ indicates more optimistic attitudes.

The ranking order results are consistent with results reported in previous literature $[8,15]$, and the results are not impacted by different values of $\lambda$. Therefore, the method proposed in this paper is applicable and effective. However, the results are not consistent with results from other literature $[4,6]$ even with appropriately-adjusted weight vectors. Analysis indicates that Zhang, et al., did not standardize cost attribute data [4]. Although Wang and Li utilized standardized cost attribute data, the definition of complement operator is different from the definition utilized in this paper [6].

\section{Conclusion}

This paper proposed improved aggregation operation rules for interval valued neutrosophic sets, and extended the generalized aggregation method to IVNSs based on the defined operators. Additionally, the appli- 
cation of the IVNS decision-making model based on IVNSGWA operator was detailed. The application of the IVNS multi-attribute decision-making method can help people make a correct decision out of available alternatives in indeterminate and inconsistent information environments in. Compared to the multiattribute decision-making method based on distance, this method maintains the integrity of information during calculation. Additionally, by choosing different parameters, decision makers can obtain ranking order results according to their own risk attitudes.

This paper only considered the attribute decision making problems with IVNS data. Considering that other data types can be expressed as IVNSs, this paper can also be easily extended to account for problems which deal with multiple data types.

\section{Acknowledgments}

The authors are indebted to anonymous reviewers for their very insightful comments and constructive suggestions, which help ameliorate the quality of this paper. This work supported by the National Research Foundation of Korea Grant funded by the Korean Government (NRF-2014S1A2A2027622), the National Natural Science Foundation of China (71471076, 71171099 and 71201071), the Joint Research of The NSFC-NRF Scientific Cooperation Program (71411140250), the Fund of Ministry of education of Humanities and Social Sciences (14YJAZH025), the Fund of China Nation Tourism Administration (15TACK003), and the Natural Science Foundation of Shandong Province (ZR2013GM003).

\section{References}

[1] F. Smarandache, A unifying field in logics. neutrosophy: Neutrosophic probability, set and logic, American Research Press, Rehoboth, 1999.
[2] H.B. Wang, F. Smarandache, Y.Q. Zhang, et al., Single valued neutrosophic sets, Multispace and Multistructure 4 (2010), 410-413.

[3] H. Wang, F. Smarandache, Y.Q. Zhang, et al., Interval neutrosophic sets and logic: Theory and applications in computing, Hexix: Arizona (2005), 6-14.

[4] H.Y. Zhang and J.Q. Wang and X.H. Chen, Interval neutrosophic sets and their application in multi-criteria decision making problems, The Scientific World Journal, In Press, http://dx.doi.org/10.1155/2014/645953

[5] I.B. Turksen, Interval valued fuzzy sets based on normal forms, Fuzzy Sets and Systems 20 (1986), 191-210.

[6] J.Q. Wang and X.E. Li, TODIM method with multivalued neutrosophic sets, Control and Decision, In Press, http://dx.doi.org/10.13195/j.kzyjc.2014.0467

[7] J. Ye, Multicriteria decision-making method using the correlation coefficient under single-value neutrosophic environment, International Journal of General Systems 42 (2013), 386-394.

[8] J. Ye, Similarity measures between interval neutrosophic sets and their applications in multicriteria decision-making, Journal of Intelligent and Fuzzy Systems 26 (2014), 165-172.

[9] J. Ye, A multicriteria decision-making method using aggregation operators for simplified neutrosophic sets, Journal of Intelligent and Fuzzy Systems 26 (2014), 2459-2466.

[10] J. Ye, Single valued neutrosophic cross-entropy for multicriteria decision making problems, Applied Mathematical Modeling 38 (2014), 1170-1175.

[11] K. Atanassov and G. Gargov, Interval-valued intuitionistic fuzzy sets, Fuzzy Sets and Systems 31 (1989), 343-349.

[12] K.T. Atanassov, Intuitionistic fuzzy sets, Fuzzy Sets and Systems 20 (1986), 87-96.

[13] L.A. Zadeh, Fuzzy sets, Information and Control 8 (1965), 338-356.

[14] P. Majumdar and S.K. Samant, On similarity and entropy of neutrosophic sets, Journal of Intelligent and Fuzzy Systems 26 (2014), 1245-1252.

[15] P.P. Chi and P.D. Liu, An extended TOPSIS method for the multiple attribute decision making problems based on interval neutrosophic set, Neutrosophic Sets and Systems 1 (2013), 63-70.

[16] R.R. Yager, Generalized OWA aggregation operators, Fuzzy Optimization and Decision Making 3 (2004), 93-107.

[17] S. Broumi and F. Smarandache, Correlation coefficient of interval neutrosophic set, Applied Mechanics and Materials 436 (2014), 511-517.

[18] V. Torra, Hesitant fuzzy sets, International Journal of Intelligent Systems 25 (2010), 529-539.

[19] Z. Xu, One method for uncertain multiple attribute decision making problems with uncertain multiplicative preference information on alternatives, Fuzzy Optimization and Decision Making 4 (2005), 131-139. 